\title{
Mutations in HS6ST1 Cause Self-Limited Delayed Puberty, in addition to Idiopathic Hypogonadotropic Hypogonadism
}

\section{Howard SR ${ }^{1}$, Poliandri $A^{1}$, Storr $\mathrm{HL}^{1}$, Metherell $\mathrm{LA}^{1}$, Cabrera $\mathrm{CP}^{2}$, Barnes $\mathrm{MR}^{2}$, Wehkalampi K${ }^{3}$, Guasti $\mathrm{L}^{1}$,}

Dunkel L ${ }^{1}{ }^{1}$ Centre for Endocrinology, ${ }^{2}$ William Harvey Research Institute, QMUL, ${ }^{3}$ Children's Hospital, Helsinki University Central Hospital and University of Helsinki

\section{Background - Puberty}

Puberty is the normal developmental stage when reproductive capacity is attained

Disturbances of pubertal timing affect over $4 \%$ of the population Deranged pubertal timing has significance for public health in view of the association between early or late puberty and an adverse cardiovascular, metabolic and cancer risk profile ${ }^{1-3}$

\section{Background - Puberty Genetics}

The timing of pubertal onset has high heritability; $60-80 \%$ of variation is genetically determined 4 - however, GWAS of age at menarche only account for $3.6-6.1 \%$ of variability ${ }^{5}$

We hypothesise that low-frequency, high or intermediate-impact variants will be enriched in a delayed puberty (DP) population at the extreme of normal pubertal timing (Figure 1).

Genetics of Delayed Puberty

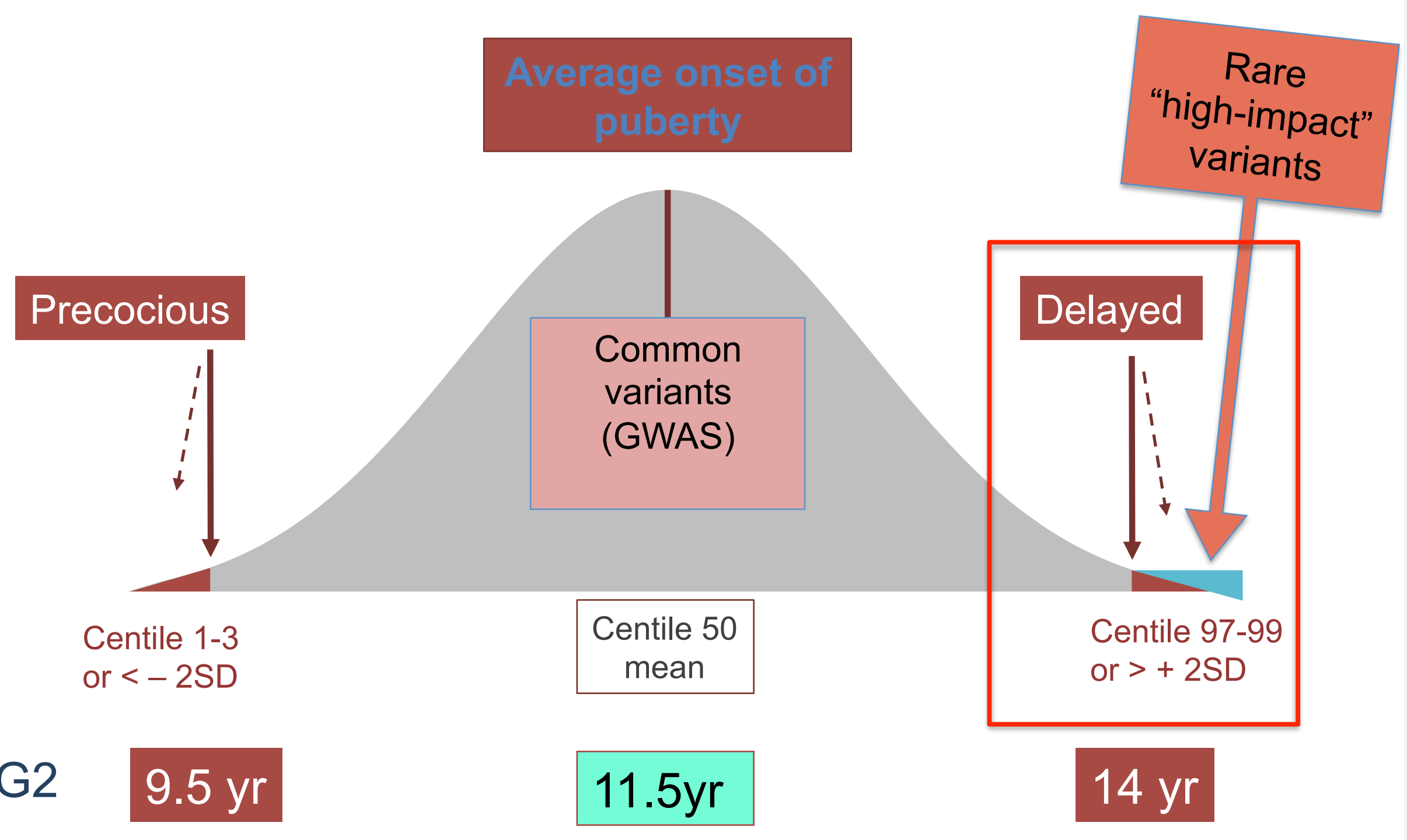

\section{Self-Limited DP}

Condition of healthy individuals with pubertal onset delayed by more than 2 standard deviations

Repeatedly been shown to cluster in families, often with AD pattern ${ }^{6}$, but pathophysiology and genetic regulation remain unclear Very limited number of rare, high impact genetic variants identified in families with both hypogonadotropic hypogonadism $(\mathrm{HH})$ and $\mathrm{DP}^{7}$

\section{Methods}

Our cohort was collected from patients seen under specialist Paediatric care from Finland between 1982-2004

Cohort contains 403 affecteds from 170 families and their unaffected relatives (total of 910 individuals)

\section{Whole Exome Sequencing Filtering Strategy}

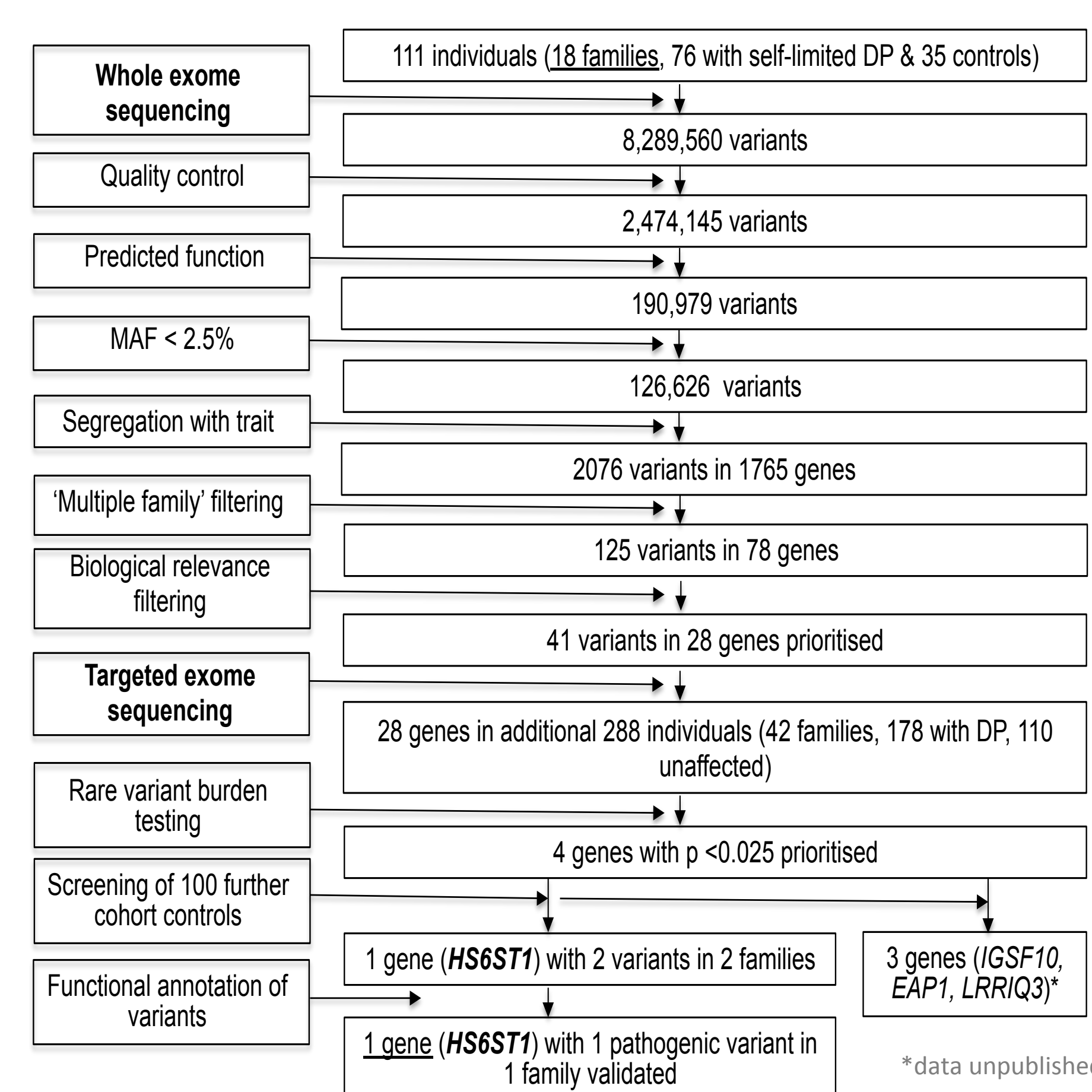

Figure 2. Our strategy for identification of new variants Began by whole exome sequencing in our 7 most extensive families.

Variants returned were filtered by a classic bioinformatics pipeline looking for rare, deleterious variants that segregate with the delayed puberty trait in each family.

Then looked for genes with variants in more than one family, with possible biological relevance to the disease, including those 20-30 genes known to be determinants in $\mathrm{HH}$

Followed by targeted resequencing in top 28 candidate genes in a further 42 families from our cohort. References 1. Widen, E., Silventoinen, K., Sovio, U. et al 2012, Diabetes Care 35(4):840-6 He, C. Zhang C. Hunter. D.J. et al. 2010. American journal of epidemiology 171:334-344. Gajdos, Z.K. Hirschhorn, J.N., and Palmert, M.R. 2009, Curr Opin Endocrinol Diabetes Obes 16:16-24. Perry J. R. Day, F. Elks, C. E. etal 2014, Nature 514(7520):92-7 Pen, J.R., De Metab 93:723-728 Zhu, J., Choa, R. E., Guo, M. H. el al 2015, JCln Endocrinol Metab 100(4):E646-54 Tornberg J., Sykiotis, G. P., Keefe, K. et al 2011, Proc Natt Acad Sci U S A 110(28):11524-9

\section{Results}

4 genes which passed rare variant burden testing included one gene known to cause $\mathrm{HH}$ : HS6ST1

1 pathogenic variant in 6 members of one family was validated

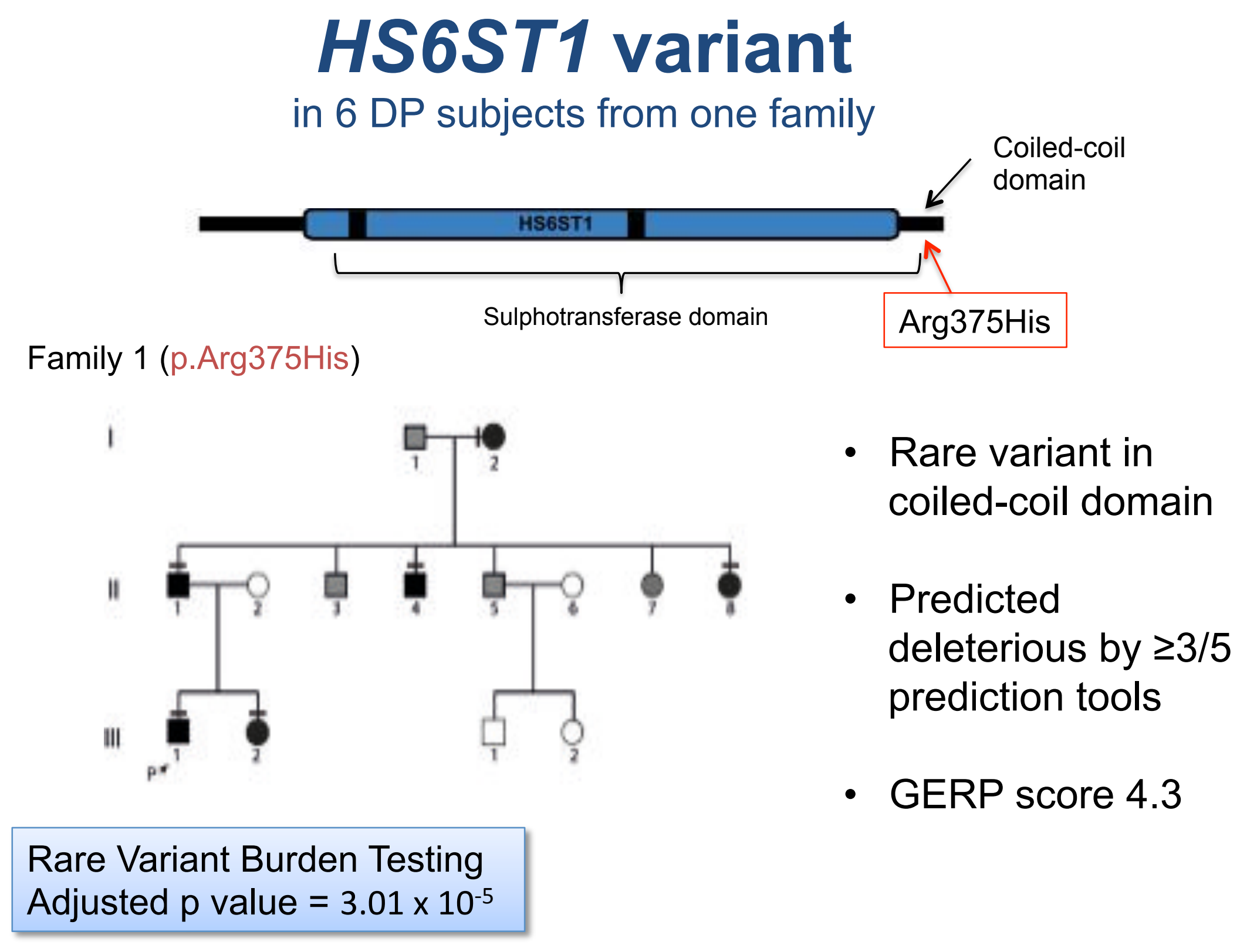

Figure 3. Details of pathogenic
variant p.Arg 375 His.
Clinical details of this family
revealed them to have typical
features of self-limited DP. The
proband case was first
investigated for growth delay at
$12.8 y r s$, at which time his bone
age was $11 y$ rs. His sister's age at
menarche was $15 y$ r; both had
normal birth weight and birth
length. Their father and paternal
uncle and aunt all had delayed
puberty with delayed linear
growth.

HS6ST1 mutations have been previously identified in up to $2 \%$ of patients with $\mathrm{IHH}^{7}$

HS6ST1 codes for an enzyme which modifies extracellular matrix components critical for normal neural branching Known to be required for the function of FGFR1 and KAL1 in vivo

Assessment of sulphotransferase activity of HS6ST1 mutant protein
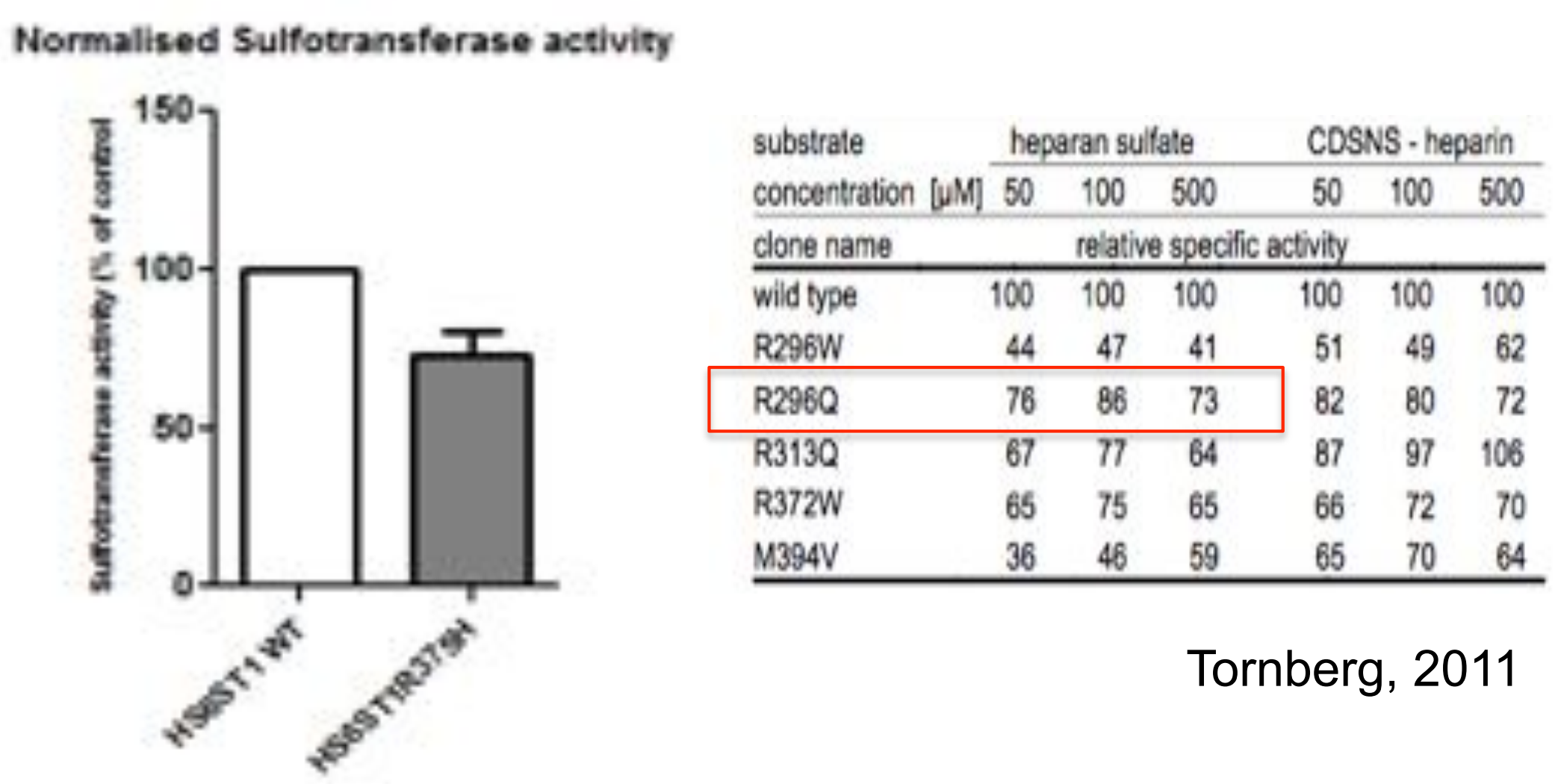

Mouse embryo studies show strong expression of HS6ST1 mRNA (in purple) from e11.5, mainly within the vomeronasal organ and olfactory epithelium (Fig. 5).

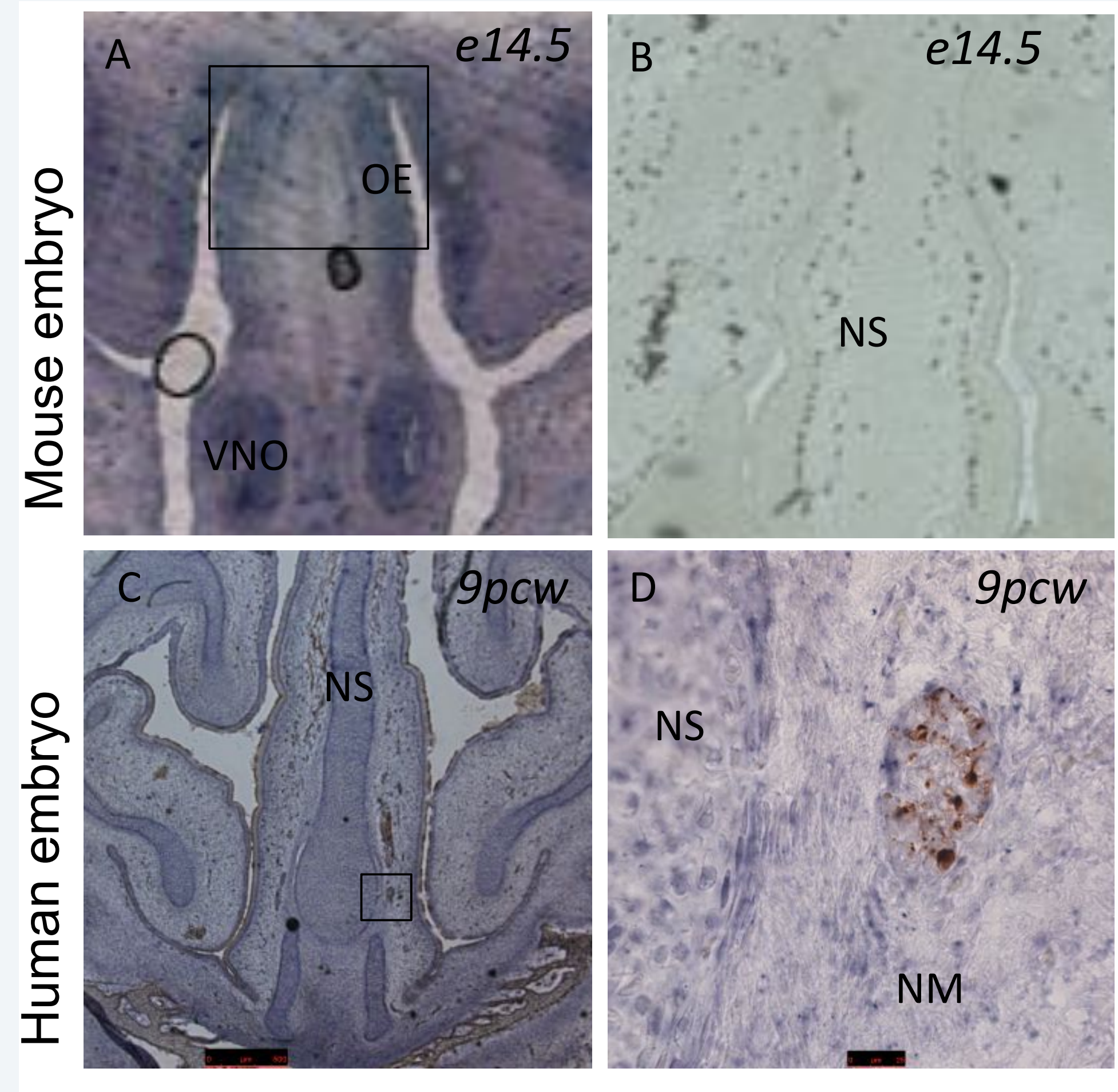

Figure 5. Expression pattern of Hs6st1 mRNA in mouse and human developing brain

Hs6st1 expression was observed from 11.5, and from e12 until e17.5 strong expression was seen in the vomeronasal organ (VNO) and olfactory epithelium (OE), as well as in the nasal mesenchyme (NM) (panel A, at e14.5).

In human 9pcw brains, HS6ST1 expression pattern was similar to that observed in mouse, with GnRH neurons interspersed in an HS6ST1-positive NM (C\&D, frontal sections). GnRH neurons are shown in brown (panels C\&D). Sense probes resulted in no specific signal (mouse probe shown in panel B, human probe not shown). NS nasal septum.

\section{Conclusions}

Mutations in HS6ST1 contribute to the phenotype of both HH and DP: -Highly conserved, deleterious variant segregating perfectly in one family with DP from our cohort

- Mutant protein has reduced sulphotransferase activity in vitro -Expression studies implicate role for HS6ST1 in developmental GnRH migration

-Supports the hypothesis that defects in $\mathrm{GnRH}$ neuronal migration and development may result in self-limited DP

-To date, there has been limited overlap between the genetic basis for $\mathrm{HH}$ and DP demonstrated from our cohort 